\title{
Characteristics of Supersonic Combustion with Hartmann- Sprenger Tube Aided Fuel Injection
}

\author{
Hongbin $\mathrm{Gu}^{1}$,Li Zhi ${ }^{2}, \mathrm{LiFei}^{3}$,Lihong Chen ${ }^{4}$,Shenglong Gu ${ }^{5}$, Xinyu Chang ${ }^{6}$ \\ Key Lab of High Temperature Gas Dynamics, \\ Institute of Mechanics, Chinese Academy of Sciences, Beijing 100190, P.R. China
}

\begin{abstract}
The TDLAS and static pressure were used to investigate the combustion of the excited ethylene jet into the supersonic flow in this article. The jet was from the Hartmann-Sprenger tube, which was put into the traditional jet device. The jet was excited at three different frequencies and compared with the base case without excitation. The results showed that the higher static pressure could be obtained in the excited cases. The excited jet didn't only take effects on the jet shear layer, but also influenced the turbulence intensity near field, and the right excited frequency can short the mixing and combusting distance. The excited jet can help reduce the combustor length.
\end{abstract}

$\begin{array}{ll}\triangle \mathrm{Q} & =\text { Heat release } \\ \triangle \mathrm{h} & =\text { enthalpy difference } \\ \mathrm{W} & =\text { mass flow rate } \\ \mathrm{Dj} & =\text { Jet diameter } \\ \mathrm{Dt} & =\text { Tube diameter } \\ \mathrm{De} & =\text { Exit diameter } \\ \mathrm{L} & =\text { Tube Length } \\ \mathrm{X} & =\text { Tube standoff } \\ \mathrm{R} & =\text { Jet Pressure ratio }\left(\mathrm{P}_{\mathrm{j} t} / \mathrm{Pa}\right) \\ V & =\text { is the velocity of the flow at the exit }\end{array}$

\section{Introduction}

Scramjet is a promising engine to provide propulsion for hypersonic vehicles. How to enhance efficient of the supersonic combustion is one of the critical topics for the improvement of scramjet performance.

The characteristic time for fuel-air mixing and combustion is only milliseconds for a scramjet operation since the velocity of the engine internal flow is over $1000 \mathrm{~m} / \mathrm{s}$. Therefore, accelerating the fuel-air mixing can enhance supersonic combustion effectively. It is known that an optimized oscillation of fuel injection could improve the fuel mixing, such as the H-S tube at high frequencies and with a simple configuration. ${ }^{[1,2,3]}$

Hartmann developed an acoustic device that generates intense wave with high frequencies. The device, which was called as the Hartmann-Sprenger tube (H-S tube) later, consists of a supersonic under-expanded jet and a coaxial resonance tube which is closed at another end. When the resonance tube is placed in the compressed region of the shock-rhombus, the H-S tube could produce rapid oscillations with large amplitude. Figure 1 gives a sketch of the H-S tube with the important parameters: the pressure ratio of the jet R, the standoff distance X, the tube length L, the diameter of the jet $\mathrm{Dj}$ and the tube Dt. Sarobia demonstrated that there are three different working modes of the $\mathrm{H}-\mathrm{S}$ tube depending on the values of the parameters as mentioned above. The first is the jet instability mode over a

\footnotetext{
${ }^{1}$ Associate Professor, Key lab of high temperature gas dynamics,

${ }^{2}$ Ph.D candidate, Key lab of high temperature gas dynamics,

${ }^{3}$ Assistant Professor, Key lab of high temperature gas dynamics,

4 Professor,

5 Graduate Student,

Key lab of high temperature gas dynamics,

6 Professor,

\author{
guhb@imech.ac.cn \\ Lizhi@imech.ac.cn \\ lifei@imech.ac.cn \\ lhchen@imech.acn \\ gushenglong@imech.ac.cn \\ xychang@imech.acn
}

Key lab of high temperature gas dynamics,
}

American Institute of Aeronautics and Astronautics 
large range of $\mathrm{X}$, excited at shear-layer initial instability frequency combined with the fundamental quarter wave of the resonance tube. The second mode, which is called as the jet regurgitant(JRG) mode, caused by the periodic swallowing and discharging of the jet flow by the resonance tube at the fundamental tube resonance frequency (f $=\mathrm{C} / 4 \mathrm{~L}$, where $\mathrm{C}$ is the sound speed in the tube). The third is called as screech mode, which is a high-frequency mode and occurred due to the formation of a normal shock in the front of the resonance tube. The strength and location of this mode was determined by the pressure ratio $\mathrm{R}$ and the standoff distance $\mathrm{X}$.

Compared with the other two modes, the JRG mode is more controllable and can produce stronger oscillations. But the frequency estimated with the equation used in Reference had a significant difference compared with the measurement, especially in the case of the small diameter of tube. Based on the experimental data, $\mathrm{Gu}^{[4]}$ modified the equation and the predicted frequencies showed a better agreement with the measurement as shown in Figure 2.

Effect of H-S tube on the mixing has been studied ${ }^{[5,6,7]}$ broadly. However, researches about the effect for supersonic combustion application were limited. This paper reported the preliminary experimental results of the supersonic combustion with $\mathrm{H}-\mathrm{S}$ tube aided fuel injection.

\section{Principle of Hartmann-Sprenger Tube Aided Fuel Injection}

Hartmann developed an acoustic device that generates intense high frequency signal. The device, which was called as the Hartmann-Sprenger tube (H-S tube) later, consists of a supersonic under-expanded jet and a co-axial resonance tube which is closed at another end. The H-S tube could produce large amplitude rapid oscillations when the resonance tube was placed in the compressed region of the shock-rhombus. There are several important parameters, which are the pressure ratio of the jet $\mathrm{R}$, the standoff distance $\mathrm{X}$, the tube length $\mathrm{L}$ and the diameter of the jet $D_{j}$ and the tube $D_{t}$, as shown in Fig.1.

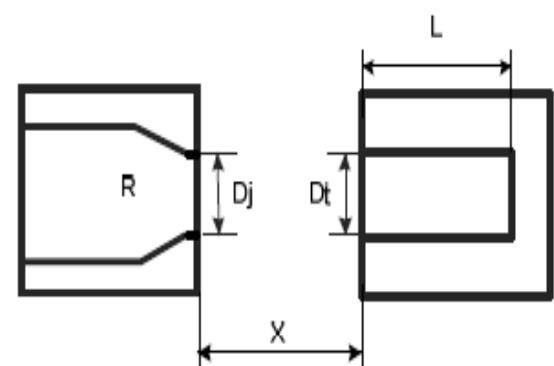

Figure 1. Sketch of the Hartmann-Sprenger tube

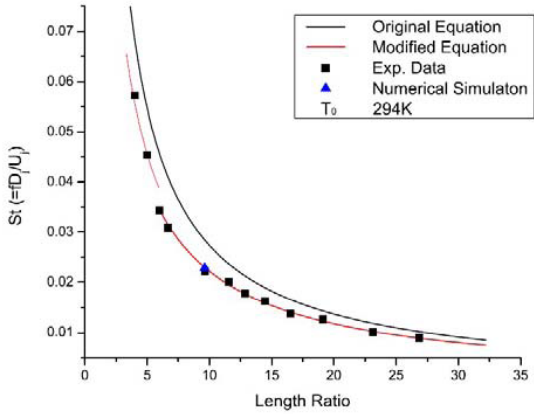

Figure 2. Comparison of estimated and measured frequency

Sarobia demonstrated that there were three different modes of the H-S tube depending on the choices of these parameters as mentioned above. The first is the jet instability mode, which was excited for subsonic jets over a large range of $X$, excited at shear-layer initial instability frequency combined with the fundamental quarter wave of the resonance tube. The second mode, which was called as the jet regurgitant(JRG) mode, occurred due to the periodic swallowing and discharging of the jet flow by the resonance tube at the fundamental tube resonance frequency $(\mathrm{f}=$ $\mathrm{C} / 4 \mathrm{~L}$, where $\mathrm{C}$ is the sound speed in the tube). The third was called as screech mode, which was a high-frequency mode and occurred due to the formation of a normal shock in the front of the resonance tube. The strength and location of this mode was governed by the pressure ratio $\mathrm{R}$ and the standoff distance $\mathrm{X}$.

Compared with the other two modes, the JRG mode is more controllable and can produce stronger oscillation. But the estimated equation of oscillation frequency in the reference had a large error compared with the measured value, especially in the case of the small diameter of tube. Therefore, $\mathrm{Gu}^{[4]}$ modified the estimated equation according to the experimental data, in order to design more accurate frequency for the case of small diameter of the resonance tube, as shown in Fig.2.

The structure of the excited jet is shown in the Fig.3, whose exit is about $5 \mathrm{~mm}$. The input to the device is $\mathrm{CO}_{2}$ flow with constant mass flow rate at a certain pressure and temperature. The output is a circular jet with fluctuating flow in the axial direction superimposed on the mean field. The H-S tube was tuned to the JRG mode while the desirable frequencies could be obtained by changing the tube length $\mathrm{L}$ with other parameters fixed. The cases were investigated under excited frequencies at $900 \mathrm{~Hz} 1.3 \mathrm{kHz}$ and $5 \mathrm{kHz}$, which compared with the base case without excitation. $^{[8]}$ 


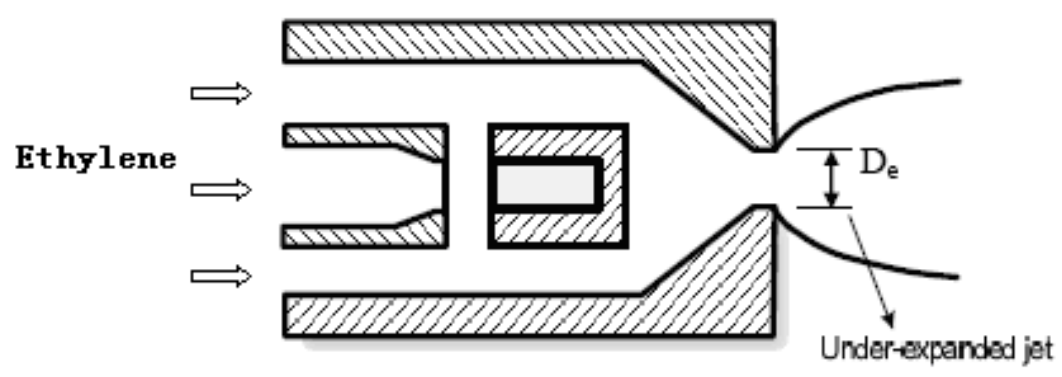

Figure 3. Sketch of the excited jet

\section{Experimental Setup}

The experiments were performed in the direct-connection supersonic combustion test facility, shown in Fig.4, in the Institute of Mechanics, Chinese Academy of Science. The air flow with high pressure and high temperature which the setup needed was supplied by the heater, and was accelerated to Mach 2.5 and 1.8 by the supersonic nozzle.

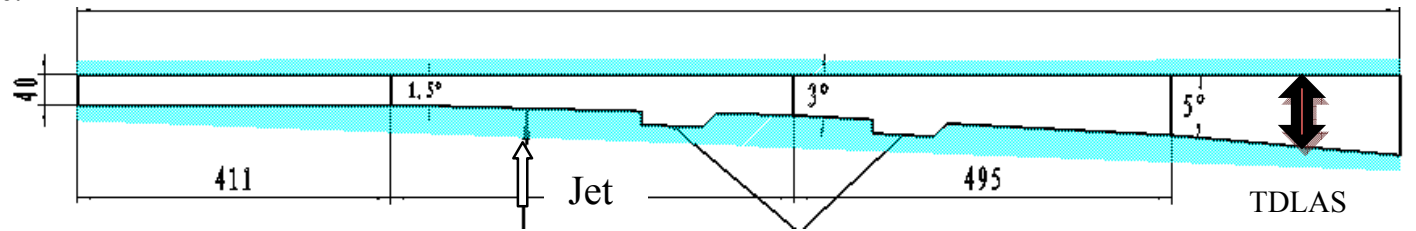

Figure 4. Configuration of combustor

Experiments were conducted in a vitiated direct-connected supersonic combustor facility. There were two inlet conditions of combustor. One was simulated at Mach number of 1.8 , the total temperatures of main flow in the experiments were $900 \mathrm{~K} \sim 930 \mathrm{~K}$, and the total pressure with $0.6 \mathrm{MPa} \pm 2 \%$. Another one was simulated at Mach number of 2.5 , the total temperatures of main flow in the experiments were $1600 \mathrm{~K} \sim 1650 \mathrm{~K}$, and the total pressure with $1.0 \mathrm{MPa} \pm 2 \%$ The total pressure .

The location of the excited jet with ethylene was shown in Fig.4, before the first cavity about $100 \mathrm{~mm}$. The combustor was expanded at single side. and had two cavities with $\mathrm{L} / \mathrm{D}=5, \mathrm{D}=18 \mathrm{~mm}$. There are about 60 pressure sensors installed along the centerline of the test section bottom wall. Schlieren system with ring knife edge and CCD at 5000fps were applied for the visualization of the shock waves and the combustion waves in the experiments.

A multi-channel Tunable Diode Laser Absorption Spectroscopy (TDLAS) system, set up at the outlet of the combustor, was used to measure temperature and velocity, and to determine the combustion efficiency. The system had two fiber coupled distributed feedback (DFB) lasers with narrow line width were used to probe three $\mathrm{H} 2 \mathrm{O}$ absorption features $(7185.597 \mathrm{~cm}-1,7444.35 \mathrm{~cm}-1$ and $7444.37 \mathrm{~cm}-1)$ by using direct absorption Time-Divisionmultiplexing (TDM) strategy at a 4-kHz scan rate. The path averaged temperature could be determined from the ratio of the integrated absorbance of the two $\mathrm{H} 2 \mathrm{O}$ transitions. Then the $\mathrm{H} 2 \mathrm{O}$ partial pressure can be determined. Gas velocity can be calculated with the measurement of the Doppler frequency shift.

\section{Results}

\section{A. Test results of Mach number 2.5}




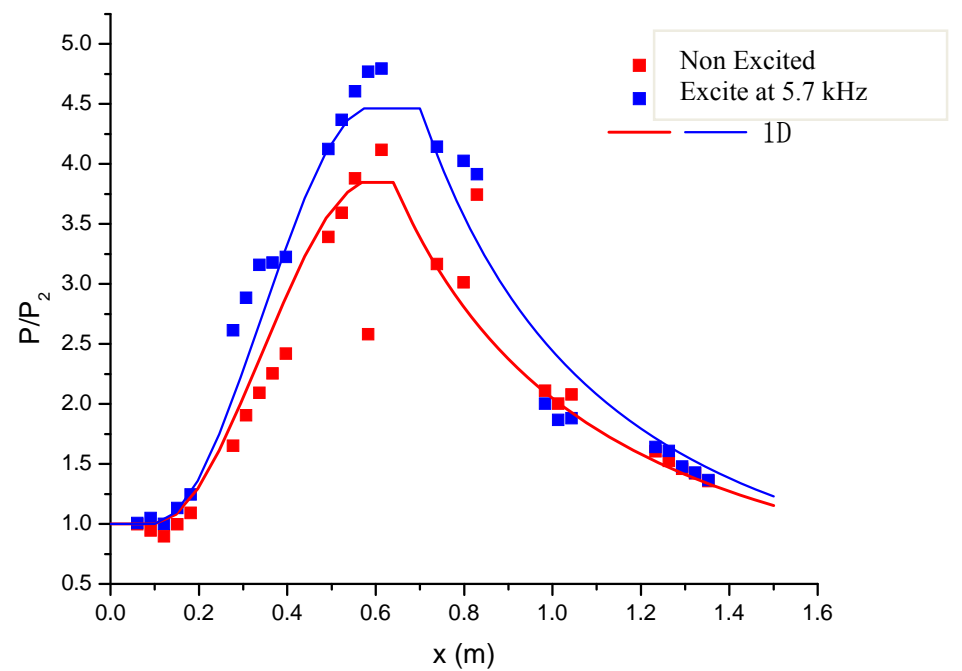

Figure 6. Dimensionless static pressure along the combustor straight wall

The dimensionless static pressure distributions along the combustor straight wall were shown in Fig.6, the isolator inlet mach number was 2.5.The X-coordinate was the length from the isolator inlet. The Y-coordinate was the dimensionless pressure normalized by the static pressure of the exiting flow of nozzle. The static pressure had a high pressure plain and decreased soon. The maximum combustion pressure of exited jet was higher $10 \%$ than non-excited jet, and pressure distribution looks more wide also. Therefore the higher combustion efficiency had been gained with excited jet. The maximum depth of unburned penetration for the excited jet was high $10 \%$ $20 \%$ than non-excited jet on same flow Mach number. In our past research, there were much large-scale structures in the jet shear layer between $5 \mathrm{D}_{\mathrm{e}} \sim 6 \mathrm{D}_{\mathrm{e}}$. whose wavelength was The depth of penetration would alternate between the big and the small with the large-scale structures existing, and there were also lots of small-scale vortexes in the jet shear layer due to the Kelvin-Helmholtz instability. The excited jet would be spread into the supersonic cross flow by those small-scale vortexes which transported by the large-scale structures. So the combustion can be proceeded more quickly, the energy release within a short length.

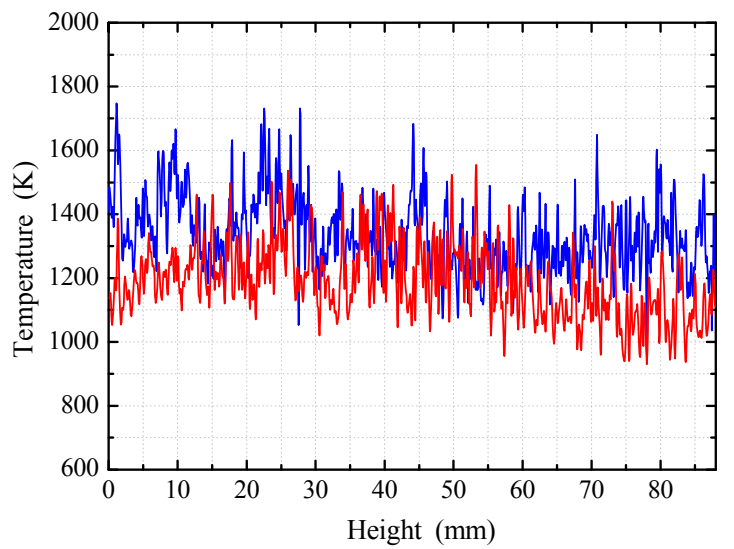

(a)

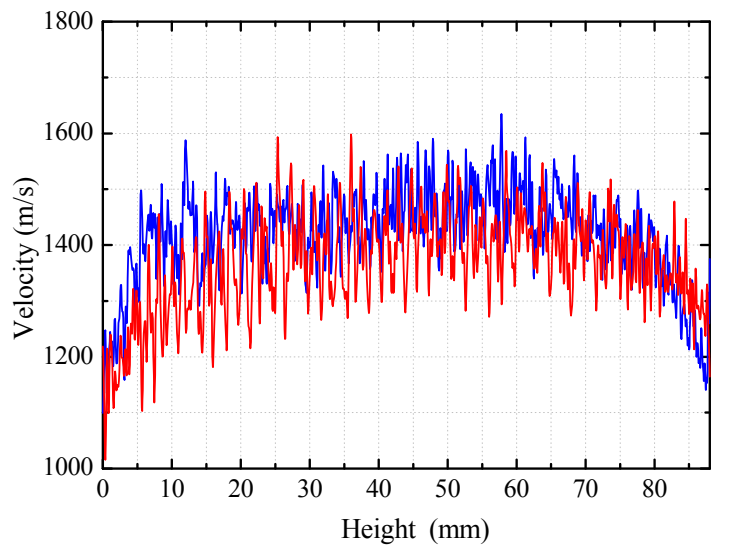

(b)

Figure 7. Temperature distribution(a) and Velocity distribution(b) along vertical location in the exit cross section of the combustor (Blue one was excited, red was non-excited)

The Temperature distribution and Velocity distribution along vertical location in the exit cross section of the jet excited and non-excited were shown in Fig. 7 . The data vibrated about $25 \%$ from minimum to maximum, though the 
data sampling frequency was about $4 \mathrm{kHz}$. It was means that the combustion had vibrated under high frequency because turbulence or unsteady flame. Obviously the blue line was higher than the red line at a majority of positions, and the velocity has the same law, so the total enthalpy of combustor with excited jet was higher than the nonexcited ones. The combustion efficiency can get from the equation at next.

$$
\begin{gathered}
\Delta Q_{c}=\Delta h+w \cdot \frac{V^{2}-V_{\infty}^{2}}{2}+\left[h_{g T}-h_{g}+w_{g} \frac{V^{2}-V_{g}^{2}}{2}\right] \\
\Delta h=\int_{0}^{H} \int_{T_{\infty}}^{T(y)} \rho V L C_{p}(T) d T d y \\
\eta_{c}=\Delta Q_{C} / \Delta Q\left(\eta_{c}=1\right)
\end{gathered}
$$

Where $\Delta Q_{c}$ is used to represent the heat release, $\Delta h$ refers to enthalpy difference, $w$ is the mass flow rate of the inflow gas, $V$ is the velocity of the flow at the exit, $V_{\infty}$ is the velocity of the flow at the entrance, $w_{g}$ is the mass flow rate of the injected fuel, $\rho$ is the mole density of the gas after combustion, $C_{p}(T)$ is specific heat capacity, $H$ is the height of the cross section at the exit of the combustor, $\mathrm{L}$ is the width of the combustor ${ }^{[9]}$. The combustion efficiency with excited was about $10 \%$ higher than without excited ones at fuel equivalence ratio equal to 0.55 .

\section{B. Test results of Mach number 1.8}

The information of Dimensionless static pressure along the combustor straight wall with different excited frequency was showed on Fig.8. The pressure had notable difference for different excited frequencies. Because mixing was better in the case of the excited jet, the deepest penetration was gained at $0.9 \mathrm{kHz}-1.3 \mathrm{kHz}$ excited case, but the most number of the large-scale structures was gained at the $5.7 \mathrm{kHz}$-excited case. The results shown that some unknown factors such as mixing properties and turbulence intensity had more effect on the combustion.

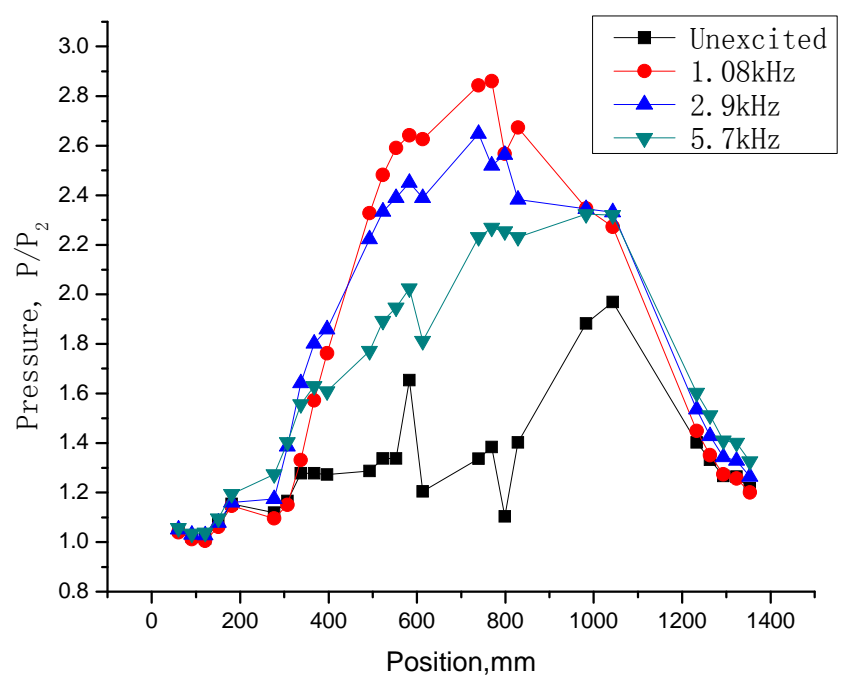

Figure 8. Dimensionless static pressure along the combustor straight wall with different excited frequency

\section{Conclusion}


The experimental investigation was carried out for the excited ethylene jet into $\mathrm{M}=2.5$ and $\mathrm{M}=1.8$ flow. According to the profile of the static pressure and TDLAS, the following conclusion could be:

1) The better mixing with less total pressure loss could be obtained in the excited cases, such as the deeper penetration, the more large-scale structures and the better combustor efficiency.

2) The excited jet didn't only take effects on the jet shear layer, but also influenced the turbulence intensity near field, and the right excited frequency can short the mixing and combusting distance.

\section{Acknowledgments}

The project is funded by the National Natural Science Foundation of China $(90305022,10525212,10621202)$.

\section{References}

[1] Sarohia V, Back L H. Experimental Investigation of Flow and Heating in a Resonance Tube. Journal of Fluid Mechanics, 1979, 94(Oct): 649-.

[2] Kastner J, Samimy M. Development and Characterization of Hartmann tube Fluidic Actuators for High Speed Control. AIAA Journal, 2002, 40(10): 1926-34.

[3] Murugappan S, Gutmark E. Parametric study of the Hartmann-Sprenger tube. Experiments in Fluids, 2005, 38(6): 813-23.

[4] S. Gu, Lihong Chen, Xinyu Chang, "Numerical Simulation and Experimental Investigation of Excited Oscillation in HS

Tube", Journal of Experiments in Fluid Mechnics, 2009.

[5] Murugappan S, Gutmark E, Carter C. Control of penetration and mixing of an excited supersonic jet into a supersonic cross stream J. Phys Fluids, 2005, 17(10).

[6] Raman G, Mills A, Othman S. Development of Powered Resonance Tube Actuators for Active Flow Control. Proceedings of ASME-FEDSM,Fluids Engineering Division. New Orleans. 2001.

[7] Kastner J, Samimy M. Development and Characterization of Hartmann tube Fluidic Actuators for High Speed Control. J. AIAA Journal, 2002, 40(10): 1926-34.

[8] GU S, CHEN L, GU H, et al. Flow-field of the excited jet and mixing in the supersonic flow; proceedings of the 16th AIAA/DLR/DGLR International Space Planes and Hypersonic Systems and Technologies Conference, October 19, 2009 October 22, 2009, Bremen, Germany, F, 2009 [C]. American Institute of Aeronautics and Astronautics Inc.

[9] Yu X L, Li F, Chen L H and Chang X Y. Spatial resolved temperature measurement based on absorption spectroscopy using a single tunable diode laser. Acta Mech Sin. Technical note.2009 